\title{
Tapping Soil Microbiota: Overlooked Underground Players in Agricultural Production
}

\author{
Yuan Wang* \\ Noble Research Institute, Ardmore, Oklahoma, USA
}

*Corresponding author: Yuan Wang, Noble Research Institute, 2510 Sam Noble Parkway, Ardmore, Oklahoma, USA, Email: “ywang@noble.org”

Received Date: September 12, 2019

Published Date: September 16, 2019

\section{Abstract}

Soil, the base for agricultural production, is constantly influenced by a variety of physical, chemical, and biological factors. Despite the abundance and diversity, soil microorganisms have been largely overlooked until recent a few years, due to the untapped functions caused by the difficulties in microbe isolation and culture. Soil microbiota, by definition, is the microbial communities that reside in specific soil niches. With the advances of next-generation sequencing technologies, more and more soil microbiomes from different soil environments were dissected genetically and functionally. These studies suggest that manipulation of soil microbiota may be one of the potential solutions for next-generation agriculture.

Keywords: Soil microbiota; Agricultural production; Plant-microbe interactions

\section{The Potentials of Harnessing Soil Microbes for Agricultural Production}

The world population has reached 7.7 billion in May 2019. The United Nations estimates that it will increase further to 11.2billion in 2100. Along with the explosion of the global population, the rapidly growing demand for food and fiber poses significant challenges to traditional agriculture. Meanwhile, overuse of chemical fertilizers and pesticides has raised many concerns for environmental pollution and food security as well. In order to solve these problems, "sustainable agriculture" and "low-input agriculture" were put forward. One of the critical strategies of both is to make use of the plant-microbe interactions to increase crop growth, fitness, and production and reduce the over-reliance on fertilizers and pesticides.

Plants and microbes have undergone coevolution for millions of years. Plant-associated microbes are so crucial to their host that it was suggested the plant and all its associated endophytic and rhizospheric microbes should be conceptualized as a "holobiont" [1]. The vast majority of plant-associated microbes come from soils. Soil, being one of the most complex ecosystems, is the habitat of various microorganisms, including bacteria, fungi, phages, protists, fauna, etc., among which bacteria and fungi are most abundant.

In the soil bacterial communities, plant growth-promoting rhizobacteria (PGPR) are a group of beneficial bacteria that competitively colonize plant roots and stimulate plant growth by a variety of mechanisms [2]. Nutritional symbiosis is commonly formed between plants and beneficial soil microbes.

One of the well-studied beneficial soil bacteria is root-nodulating bacteria, the Rhizobia. Rhizobia bacteria specifically infect roots of the plants belonging to Leguminaceae family. Under nitrogen deficiency, legumes secrete flavonoids and other substances as specific signals for the recruitment of rhizobia from soils. The infection of rhizobia induces the organogenesis of root nodules, where nitrogen is fixed by rhizobia and converted to ammonium ions, a plant-available nitrogen source [3]. A typical example of plant-fungus nutritional symbiosis is arbuscular-mycorrhizal fungi (AMF). AMF invade the cortical root cells of the vast majority of angiosperms plants and form symbionts inside of the host plants [4]. Meanwhile, the extensive mycorrhizal hyphae of AMF extend into the soils to acquire more nutrients such as phosphorus and nitrogen for the plants [5]. In turn, AMF receives carbon from the host plants.

The beneficial effects of such microorganisms are significant for plant performance, agricultural production, and soil health. However, these well-studied microbes are just the tip of the iceberg, while the functions of the vast majority of the soil microorganisms have not been tapped yet. Traditionally, the characterization and functional study of a microorganism replied on the isolation and 
culture of the microorganism. However, less than one percent of the soil microorganisms can be cultured in vitro.

Moreover, the influence of soil microbes on plants do not exist as an individual, but as a complex microbial community. Soil microbial species are diverse and abundant, with millions to billions of microbes in one gram of soil. In a specific soil niche, thousands of species of soil microbes with varied abundances form a network through complex interactions, making it a whole as the microbial community, or microbiota. Investigating the effects of soil microbiota on plants in a specific environmental condition is unusually challenging.

With that said, some critical questions need to be addressed: Who is there in a specific soil niche? How are the soil microorganisms influenced by soil physical and chemical characteristics? How does a microbial community comprising of thousands of microorganisms perform specific functions to serve the plants in a particular environment? With the advances of next-generation sequencing technologies, the sequencing and analysis of the microbiome, i.e., all the genomes in one microbiota, have become a new approach to explore the function of soil microbiota at the community level.

\section{Appropriate Approaches for Investigating Soil Microbiomes}

\section{Sampling strategies for soil microbiomes}

Soil sampling strategies vary depending on the purpose of the study. Most researches are currently focused on natural soils and rhizosphere soils. Sampling natural soils are simple and easy to operate, but attention needs to be paid to soil heterogeneity, and microbial communities are susceptible to the micro-environment, so multiple sampling is required for better spatial representation. Sampling rhizosphere soil is more complicated. First, the current study does not define the definition of rhizosphere soil. Some studies are broadly defined, such as planting plants in pots. After a period of plant growth, the roots are more widely distributed in the pot, i.e., the soil in the pot is considered to be the rhizosphere soil. Most of the research now tends to be more strictly rhizosphere soil, that is, about $2-3 \mathrm{~mm}$ thick around the roots of plants, firmly attached to the soil layer on the roots. Soil sampling in these studies required the collection of plant roots to remove loosely attached soil from the roots, after which most of the buffer soil was used to collect the rhizosphere soil. Of course, the complexity of plant roots may also lead to significantly different rhizosphere microbial communities at different developmental stages and different parts, but the current rhizosphere microbial community research has not yet reached this resolution.

Besides, the dynamic changes of soil microbial communities have also attracted more and more attentions [6]. In the initial soil microbial community study, due to the practical operational difficulties of soil sampling in the field environment, many studies only sampled at just one time point. However, subsequent studies have shown that the microbial community of natural soils varies with seasons and years, while the rhizosphere soil changes dynamically with the growth and development of plants during the growing season. More and more research results show that sampling at one time point is only a snapshot of the soil microbial community, and the dynamic pattern of soil microbial community is more meaningful compared to a single snapshot.

\section{The sequencing-based methodologies for soil microbiomes}

At present, amplicon sequencing and shotgun metagenomics are the two commonly used approaches for soil microbiome analysis. Soil microbiota contains thousands to tens of thousands of microorganisms, including bacteria, fungi, protists, microfauna, etc. Amplicon sequencing for community profiling is an excellent way to simplify the complexity of soil microbiota. Amplicon sequencing is based on PCR amplification of phylogenetic markers and was used to characterize the taxonomic composition and phylogenetic diversity of metagenome samples [7]. Phylogenetic marker is a DNA fragment or gene locus, which meets specific criteria to be used in species identification and phylogenetic reconstructions. The requirements for a phylogenetic marker include:

having no or predictable variation within a given species, having detectable differences between species, and being available for most or all species of a genus. Commonly used phylogenetic markers are 16S rRNA genes for bacteria and archaea, Internal Transcribed Spacer (ITS) region for fungi and 18S rRNA genes for eukaryotes. Amplicon sequencing of soil microbiomes answered the question "who is there."

Shotgun metagenomics analyzes all functional genes of soil microbiota compared to amplicon sequencing, which can only interpret the community profiling based on phylogenetic markers. Thus, shotgun metagenomics provides a much more comprehensive understanding of the potential functions of soil microbiota. In shotgun metagenomics, microbial DNA extracted from soil samples, i.e., soil metagenomes, was digested into DNA fragments and then sequenced at high resolution to retrieve all the sequence information of the soil metagenome [8]. Due to the characteristics of microbial genes, including diversity, homology, and redundancy, subsequent sequence analysis, gene binning, etc. require the assistance of more powerful bioinformatics tools. At present, the methodologies for shotgun metagenomics have been well developed and adopted by many researchers. It should be noted that although shotgun metagenomics can analyze the presence or absence of the microbial functional genes in the soil microbiota, this is merely an analysis at DNA level and can only address the question "what is the potential functions of the soil microbiota". As for whether these functional genes are expressed and how is the level of expression, meta-transcriptomic studies at RNA level need to be performed, which is even more challenging and still in its infancy.

\section{Prospects for the application of soil microbiota in agricultural production}

Harnessing beneficial soil microbes to facilitate crop growth and productivity is one of the keys for next-generation agriculture. However, the domestication and the breeding of modern crop 
cultivars which aimed at maximized yield with the use of chemical fertilizers and pesticides, have affected the associated microbiota and compromised certain beneficial soil microorganisms [9]. A comprehensive understanding of soil microbiota in the agricultural system is required for the optimization of plant-microbiota interactions to benefit agricultural production.

Current advances in soil microbiota shed light on the potentials of the microbiota-based breeding strategy. First, several studies have successfully selected a particular soil microbiota to modify plant traits [10]. Further, the soil microbiota structure was shown to be heritable between generations [11]. Thus, it is possible to prepare the field microbiologically for the optimum crop. Besides, the capacity and efficiency of plants in selecting beneficial microbiota may be an important trait that can be potentially exploited for breeding purpose.

To design the microbiota-based breeding strategy, first of all, we need in-depth dissections of rhizosphere soil microbiota in crop plants, including following the spatial and temporal changes of soil microbiota with high resolutions. Further, understanding how plant productivity and fitness traits are synergistically controlled by plant genomes and plant-associated microbiomes will contribute to the design of future crops that, in combination with dedicated microbial agriculture, can sustainably enhance crop production. In the past few years, advances in plant microbiomes provided essential insights into processes underlying structure and assembly of the microbial community. There is still numerous "black box," and knowledge gaps are awaiting us to discover: characterization of rhizosphere signaling compounds, mechanisms involved in the molecular dialog between plant roots and the rhizosphere microbiome, identification of crop key genes controlling beneficial microbes' root colonization, etc. All these are crucial issues for crop breeding to be considered.

\section{Acknowledgement}

This work is supported by the U.S. Department of Energy Office of Science, Office of Biological and Environmental Research
Genomic Science program under Award Number DE-SC0014079 to UC Berkeley, Noble Research Institute, the University of Oklahoma, Lawrence Livermore National Laboratory and Lawrence Berkeley National Laboratory.

\section{Conflicts of Interest}

No conflict of interest.

\section{References}

1. Guerrero R, Margulis L, Berlanga M (2013) Symbiogenesis the holobiont as a unit of evolution. Int Microbiol 16(30): 133-143.

2. Pieterse CMJ, De Jonge R, Berendsen RL (2016) The Soil-Borne Supremacy. Trends in Plant Science 21(3): 171-173.

3. Oldroyd GE, Murray JD, Poole PS, Downie JA (2011) The rules of engagement in the legume-rhizobial symbiosis. Annu Rev Genet 5: 11944.

4. Brundrett MC (2009) Mycorrhizal associations and other means of nutrition of vascular plants: understanding the global diversity of host plants by resolving conflicting information and developing reliable means of diagnosis. Plant and Soil 320: 37-77.

5. Smith SE, Read DJ (2010) Mycorrhizal symbiosis. Academic press

6. Shi S, Nuccio E, Herman DJ, Rijkers R, Estera K et al. (2015) Successional trajectories of rhizosphere bacterial communities over consecutive seasons. M Bio 6: e00746-00715.

7. Kozich JJ, Westcott SL, Baxter NT, Highlander SK, Schloss PD (2013) Development of a dual-index sequencing strategy and curation pipeline for analyzing amplicon sequence data on the MiSeq Illumina sequencing platform. Appl Environ Microbiol 79: 5112-5120.

8. Quince C, Walker AW, Simpson JT, Loman NJ, Segata N (2017) Shotgun metagenomics, from sampling to analysis. Nat Biotechnol 35(9): 833844.

9. Bulgarelli D, Garrido Oter R, Münch Philipp C, Weiman A, Dröge J et al. (2015) Structure and Function of the Bacterial Root Microbiota in Wild and Domesticated Barley. Cell Host Microbe 17(3): 392-403.

10. Panke Buisse K, Poole AC, Goodrich JK, Ley RE, Kao Kniffin J (2014) Selection on soil microbiomes reveals reproducible impacts on plant function. The Isme Journal 9: 980.

11. Donn S, Kirkegaard JA, Perera G, Richardson AE, Watt M (2015) Evolution of bacterial communities in the wheat crop rhizosphere. Environmental microbiology 17(3): 610-621. 